\title{
Technical Aspects of Web Photography as a Medium of Tourism Development
}

\author{
Petra Ptiček, Ivana Žganjar, Miroslav Mikota*, Mile Matijević
}

\begin{abstract}
Information and communication technology is an important factor for national, regional and local sustainable tourism development according to the long-term Croatian national strategic plan. New forms of information, such as web sites; new media, materials, political and social change, all influence tourists' decisions when choosing specific destinations. The aim of this research is to determine, based on the analysis of the tourism media campaign, the relationship between new communication trends and the application of photography as a medium that influences the experience when choosing a destination and the importance of crucial information factors on web pages based on their technical and visual characteristics.
\end{abstract}

Keywords: color; photography; tourism development; web pages

\section{INTRODUCTION}

Tourism relies on the application of new technologies' modern trends, innovations, and software solutions in order to meet the individual needs of tourists. The influence of changes in technology is reflected in the decision making regarding the promoted destination because of the tourists' need to choose the desired destination on their own. Therefore, marketing communication in the digital world is focused on creating a presence, a relationship and added value for consumers and services in tourism. The basic concept of integrated marketing communication is aimed at encouraging the user to go to the desired destination relying on the promotional message. With the development of new media, new promotion services are emerging, and the emphasis is on multimedia and interactivity. Combining services and applying the appropriate marketing strategy is reflected in making appropriate decisions. Photography and fast loading of content on tourist websites are an important element of advertising. Web sites have the ability to spread information in a very short time. Consequently, web sites in tourism have changed the way people research, gather information, make decisions and share their experiences. When choosing their destination, tourists search the internet to explore offers, accommodation, and useful tips from other tourists. In order to connect through web sites, in the most appropriate, informative, and interesting way, photographic images of the desired destinations have proven to be a key element in tourism promotion as a means of information exchange [1]. In the digital age, the importance of the visual comes to the fore because it will occupy the observer and involve them emotionally. Photography is necessary for the presentation of products, ideas, thoughts, services, and it attracts attention and directs the user to make decisions about their tourist experience. Today, when photographing advertising photography, the photographer is expected to have a wide range of knowledge, experience and fresh ideas. It is important to emphasize that advertising photography goes through extensive editing $[2,3]$. Photography's indirect purpose is to realize the sale of the desired service. Consumers spend $80 \%$ of their time on the perception of photography, and $20 \%$ on the content of a text message, so it is necessary to realize the idea of the photographer on a perceptual level [4]. Perception is the process of receiving, processing and storing stimuli that builds an impression of a service. There is no simpler and more elegant way to influence the viewer other than using photography [5]. The goal of photography is to evoke emotions and feelings in the viewer that describe the product and service. Clear communication between the photographer and the viewer is necessary for photography to be successful [6]. Photography provides endless possibilities for expressing and experiencing emotions. That is precisely the reason why photography is an indispensable element when using visual media for the purpose of advertising in tourism. Photography, especially portrait photographic images, attracts attention, transmits information and enhances communication and understanding of the web site's tourist offerings, $[7,8]$. Quality tourist web pages based on visual elements and professional photography can create a better user experience and spur the development of tourism. Previous research on the analysis of photographic images of human faces shows that their use contributes to the informativeness of the website. Research has shown that a smiling face, the face facing the camera, authenticity, and simple backgrounds are elements which draw attention to the content of web pages [9]. For this reason, the authors explore the visual content under the technical characteristics of web pages and that includes photography as a design element in a digital environment. Preliminary research was based on the presence of these elements: color, movement, photography and logos, and the use of symbols and their characteristics. Following the preliminary results, which support the hypothesis of the importance and role of photography as an element that attracts and retains the user on the website and increases the amount of information, the authors in this scientific research analyze photography as one of the most important multimedia components reflected in the tourists' destination selection based on the definition of the technical characteristics of the human face.

\section{THE EXPERIMENT}

In accordance with the web-based national presentation of tourism in the Republic of Croatia, the authors conducted a research on eleven websites defined at the national level. 
The results of the scientific research show that facial photography affects the quality of the tourist website, creating a better user experience, Fig. 1.

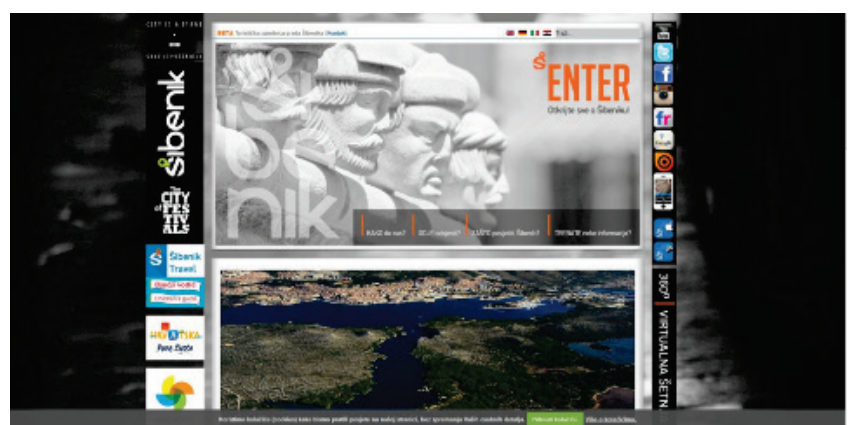

Figure 1 The sample "Sibenik" was analyzed as a whole according to the observed contents of the website: A-social networks; B- text ; C-central photography of the face; D-logo; E-Šibenik logo; F-navigation; G-picture above. Source: https://www.sibenik-tourism.hr

The statistical analysis in this phase of the research included all data from the empirical part of the research analyzed by the method of descriptive statistics. The survey, conducted on 150 participants, (75 male, 75 female participants, the average age 25), established a subjective assessment of the quality of the seen content based on the information and guidelines for tourism development while taking into consideration the user's needs. The statistical package STATISTICA 12.0 was used for statistical data processing. Samples were displayed on an ASUS PA27AC, HDR monitor and viewed at a distance of $650 \mathrm{~mm}$ from the screen. Observer reactions and the number of fixations required to perceive information were determined and measured using the Tobii Eye Tracker X60. The software used to conduct the test was Tobii Studio 3.2.1. The observers were placed in front of the monitor which was located in a separate room without additional lighting, according to the standard for graphic technology and professional photography ISO 3664:2009. At the beginning of each individual experiment, the respondents were explained the task and the content displayed on the monitor. At the end of the survey, the respondents filled out a questionnaire. During the experiment, using the Tobii Eye Tracker X60, the movement of the gaze was recorded on eleven samples, the time and number of fixations that the subject needed to notice the information were measured. In order to establish the way in which the participants viewed the samples, certain areas of interest were defined on each of the eleven samples according to their visual characteristics in such a way that each individual graphic element was considered a separate area of interest.

The analysis of the characterization of web page elements as an information and communication tool, and the statistical parameters of the respondents' evaluation of each web page shows that among the technical characteristics aimed at optimizing network tools intended for promotional activities, "Google Chrome", was rated as best, followed by "Mozilla Firefox", "Internet Explorer" and "Opera". Among social networks that enable interaction and networking, "YouTube" was rated as best, then "Facebook", then
"Instagram" and "Twitter". The best rated element of visual features is photography, followed by dominant color, logo, and movement. The best rated product from the development perspective is the production group "sun and sea", followed by adventure and sports tourism, cultural tourism, gastronomy and oenology, nautical tourism, rural mountain tourism, ecotourism, cycling, and health tourism, while the lowest rated were golf and business visits.

The stated characteristics of the content of web pages as an information and communication tool in relation to the technical characteristics, visual characteristics and the presence of content from the perspective of development on web site sample "Šibenik" included areas of interest of respondents (Fig. 2). It also included the following design elements in the digital environment: central photography faces, picture 2, logo, $\log 02$, social networks, striking text, navigation bar, in relation to the samples that include the following areas of interest: picture 3 , picture 4 , $\log$ o 3 , virtual walk / application, multilingualism, search engine.

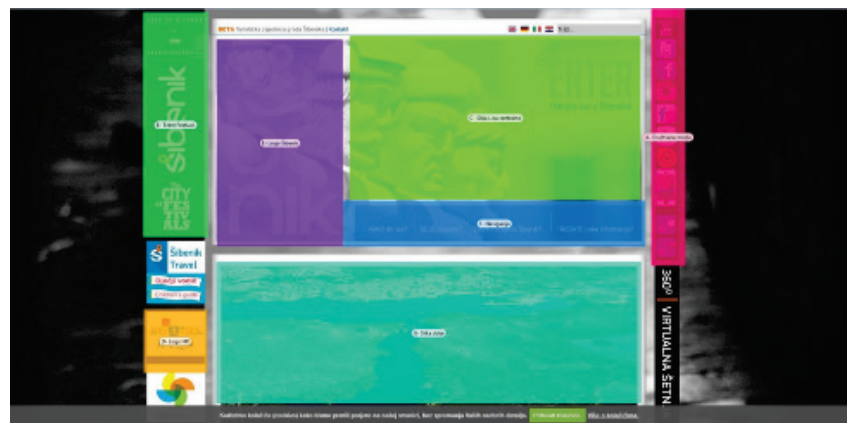

Figure 2 Areas of interest of all respondents for the sample "Šibenik". Source: https://www.sibenik-tourism.hr

The results show that participants who were offered visual content of web site samples based on web tools that offer more choices on how to use the content, rated it more accessible, informative and credible when planning and organizing trips, and the analysis based on Tobii Eye Tracker X60 showed that the website sample "Šibenik" includes the presence of visual information of new network tools and confirms the presence of visual information through seven areas of interest of the total observed. When viewing the sample, the main points of interest, visual entries of the participants to the web page are the central photography of the face, picture $2, \operatorname{logo}$, logo 2, social networks, striking text, navigation bar. The achievement of the goals defined on national level is based on the assumption that promotional activities tailored to end users, based on ease of access to information and credibility, positively reflect on their selection and decision-making process with direct impact on the economy. The analysis of the sample "Šibenik" based on application of new online tools for promotional activities confirms the hypothesis of contribution to the development of tourism as a strategic branch of the economy. Based on the analysis of the portrait photographic images on the referent website, the reference file size of the portrait photographic image digital record of $28.8 \mathrm{M}$ was determined as the original digital record size. Digital biometric portrait photographic 
image obtained by shooting with the digital Leica format Canon Eos 5DSR camera with the maximum resolution of 50 MP in Adobe RGB color space with correct white balance and correct exposure elements (exposure time 1/60 sec, lens aperture 5.6) determined by TTL for sensitivity of 100/21 ISO was taken. The TTL system was used segmentally on the skin color of the face. The Kaiser 1000 halogen reflector was used as the light source. The photographic image was saved in the finest JPG format (12) and loaded in Adobe Photoshop 2020 program where it was converted to the reproducible sRGB color space, and the file size was reduced from recorded to the reference size of $28.8 \mathrm{M}$. The photographic image was saved in the finest JPEG format and defined as the original. Defined original photographic image was degraded for the purposes of research by using the tools of Adobe Photoshop 2020 program.

The first degradation was performed by simulating the exposure change in the step of decreasing and increasing $1 / 3$ of the lens aperture in the range of \pm 1 lens aperture.

The second degradation was performed by changing the saturation in steps of \pm 5 in the range \pm 15 .

The third degradation was performed by changing the color temperature by simulating the application of correction filters: WF (85), WF (LBA), WF (81), CF (80), CF (LBB), CF (82).

The fourth degradation was obtained by simulating the decrease in sharpness in the range of 10-60 with the change step of 10. The fifth degradation was obtained by saving the digital record of the original photographic image in four fineness of JPG records: low (1), medium (5), high (8), maximum (12).

Degradations 1-3 were performed in the same way on the Adobe Camera Raw color table, and degradations 4 and 5 on the ISO 12233 resolution table, which was previously, as the original resolution table, reduced from the file size $47,5 \mathrm{MP}$ to the reference $28,8 \mathrm{M}$. The original and degraded photographic images were evaluated by six experts in the field of photographic images by the simultaneous haploscopic (S) and memory (M) method of comparison in controlled conditions according to the standard for graphic technology and professional photography ISO 3664:2009. For the evaluation ASUS PA27AC calibrated monitor was used. Experts rated the degraded photographic images on the Lickert scale of 1-4 where rating 1 means completely unacceptable, 2 unacceptable, 3 acceptable and 4 completely acceptable degradation. Degraded photographic images were evaluated by experts according to the criteria of acceptability of changes in exposure, saturation, color temperature and sharpness (Fig. 3). For degraded color tables (degradation 13 ), using Adobe Photoshop 2020, L* a* b* values of standard light skin, dark skin and achromatic colors were determined, as well as average $L^{*} a^{*} b^{*}$ color values of average table color. For all observed colors, the total color change $\Delta E_{00}$ was determined. On the degraded resolution tables (degradation $5,6)$, the resolution of the lines horizontally and vertically according to the image height ( $\mathrm{LW} / \mathrm{PH})$ was determined. a)
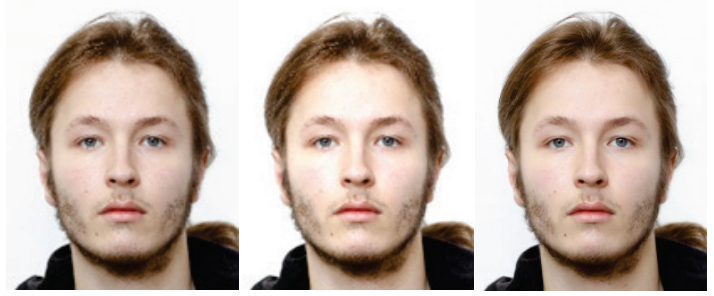

b)
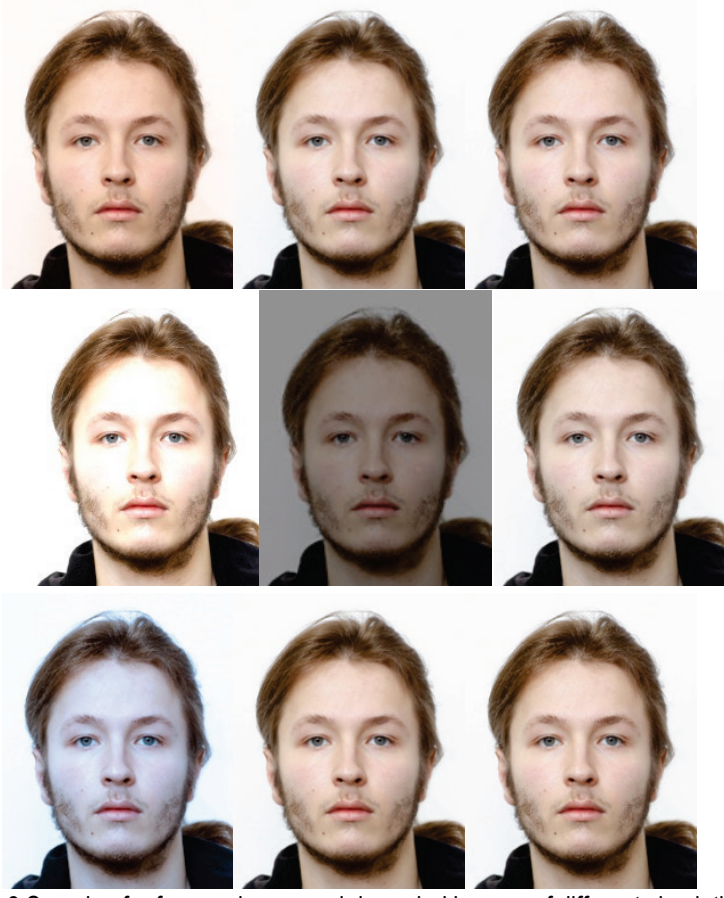

Figure 3 Sample of reference image and degraded images of different simulations: a) completely acceptable b) completely unacceptable

\section{REASERSCH RESULTS WITH DISCUSSION}

The results of the empirical research based on the application of eye tracking technology are shown in Tab. 1 and Fig. 4.

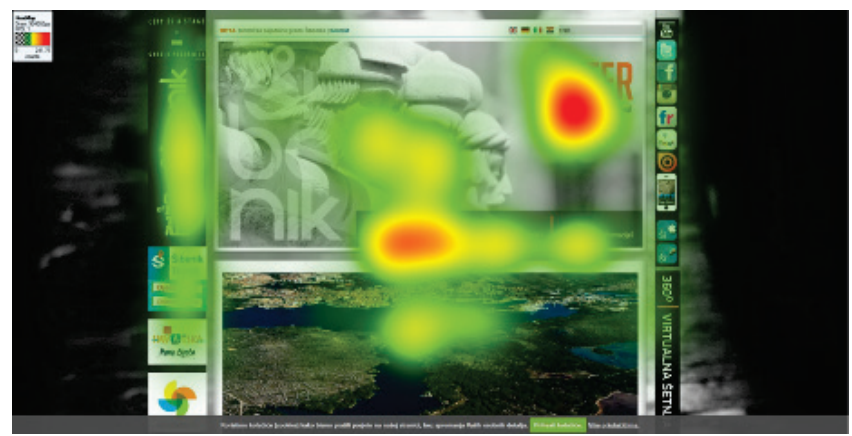

Figure 4 Thermal maps of the total value of all participants for the sample "Šibenik". Source: https://www.sibenik-tourism.hr

Statistical parameters for the variables Time to first fixation, Total duration of fixation, Fixation count and Visit duration grouped according to the observed contents of the sample "Sibenik" are shown in Tab. 1. Time to first fixation shows a wide range of shortest time from only $0.63 \mathrm{~s}$ for content C-central photo of face to $4.90 \mathrm{~s}$ for content D-logo Hrvatska. The total duration of fixation, the fixation count 
and the visit duration is the longest for the content of the Ccentral photography of the face (Fig. 2).

The results of the experts' estimation of the acceptability of the degradation of the portrait photographic image by simulating the changes of exposure, saturation and applying corrective filters changes are shown in Tabs. 2, 3 and 4.

\begin{tabular}{|c|c|c|c|c|c|c|c|c|}
\hline \multirow[b]{2}{*}{ Variables } & \multirow{2}{*}{$\begin{array}{c}\text { Statistical } \\
\text { parameters }\end{array}$} & \multicolumn{7}{|c|}{ Content } \\
\hline & & & & $C$ & & $\mathrm{~F}$ & $F$ & \\
\hline \multirow{6}{*}{ 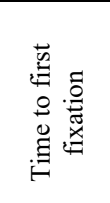 } & $\overline{\mathrm{X}}$ & 4,61 & 3,24 & 0,63 & 4,90 & 1,78 & 1,96 & 2,19 \\
\hline & SD & 2,60 & 2,33 & 1,03 & 2,19 & 1,95 & 1,94 & 2,13 \\
\hline & SE & 0,34 & 0,22 & 0,08 & 0,31 & 0,19 & 0,17 & 0,18 \\
\hline & Min. & 0,00 & 0,00 & 0,00 & 0,00 & 0,00 & 0,00 & 0,00 \\
\hline & Max. & 7,99 & 7,98 & 6,06 & 8,04 & 7,10 & 7,98 & 7,56 \\
\hline & $\mathrm{M}$ & 5,30 & 3,33 & 0,32 & 5,53 & 0,76 & 1,49 & 1,87 \\
\hline \multirow{6}{*}{ 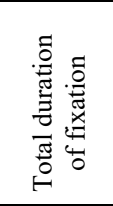 } & $\overline{\mathrm{X}}$ & 0,59 & 1,06 & 2,51 & 0,36 & 0,69 & 1,55 & 1,33 \\
\hline & SD & 0,38 & 0,75 & 1,40 & 0,19 & 0,54 & 1,28 & 1,06 \\
\hline & SE & 0,05 & 0,07 & 0,12 & 0,03 & 0,05 & 0,11 & 0,09 \\
\hline & Min. & 0,06 & 0,07 & 0,25 & 0,01 & 0,10 & 0,06 & 0,03 \\
\hline & Max. & 1,60 & 3,98 & 8,05 & 1,12 & 2,71 & 7,20 & 5,49 \\
\hline & $\mathrm{M}$ & 0,51 & 0,87 & 2,22 & 0,32 & 0,52 & 1,27 & 1,03 \\
\hline \multirow{6}{*}{ 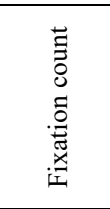 } & $\overline{\mathrm{X}}$ & 2,07 & 3,76 & 8,24 & 1,21 & 2,59 & 5,04 & 4,42 \\
\hline & SD & 1,34 & 2,33 & 3,55 & 0,41 & 1,71 & 3,23 & 3,23 \\
\hline & SE & 0,18 & 0,22 & 0,29 & 0,06 & 0,16 & 0,28 & 0,28 \\
\hline & Min. & 1,00 & 1,00 & 1,00 & 1,00 & 1,00 & 1,00 & 1,00 \\
\hline & Max. & 6,00 & 14,00 & 21,00 & 2,00 & 8,00 & 14,00 & 17,00 \\
\hline & $\mathrm{M}$ & 2,00 & 3,00 & 8,00 & 1,00 & 2,00 & 5,00 & 3,00 \\
\hline \multirow{6}{*}{ 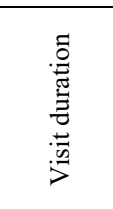 } & $\overline{\mathrm{X}}$ & 0,55 & 0,84 & 1,11 & 0,35 & 0,40 & 0,87 & 0,70 \\
\hline & SD & 0,39 & 0,60 & 0,91 & 0,19 & 0,31 & 0,84 & 0,49 \\
\hline & SE & 0,05 & 0,06 & 0,07 & 0,03 & 0,03 & 0,07 & 0,04 \\
\hline & Min. & 0,06 & 0,07 & 0,24 & 0,01 & 0,10 & 0,06 & 0,03 \\
\hline & Max. & 1,60 & 4,15 & 8,05 & 1,12 & 2,71 & 4,61 & 2,83 \\
\hline & $\mathrm{M}$ & 0,40 & 0,70 & 0,87 & 0,30 & 0,30 & 0,57 & 0,58 \\
\hline
\end{tabular}

Table 2 Experts' estimation for degradation in exposure

\begin{tabular}{|c|c|c|c|c|c|c|}
\hline Method & $+0,33$ & $-0,33$ & $+0,66$ & $-0,66$ & +1 & -1 \\
\hline $\mathrm{M}$ & 4 & 3,2 & 1,5 & 2,2 & 1 & 1,3 \\
\hline $\mathrm{S}$ & 4 & 3,1 & 1,6 & 2,3 & 1 & 1,2 \\
\hline
\end{tabular}

Table 3 Experts' estimation for degradation in saturation

\begin{tabular}{|c|c|c|c|c|c|c|}
\hline Method & +5 & -5 & +10 & -10 & +15 & -15 \\
\hline M & 3,8 & 3,7 & 3 & 3 & 2,3 & 3 \\
\hline S & 3,7 & 3,6 & 3 & 3 & 2,2 & 3 \\
\hline
\end{tabular}

Table 4 Experts' estimation for degradation in color temperature

\begin{tabular}{|c|c|c|c|c|c|c|}
\hline Method & $\begin{array}{c}\text { Warm } \\
\text { LBA }\end{array}$ & Warm 81 & Warm 85 & Cool 80 & Cool 82 & $\begin{array}{c}\text { Cool } \\
\text { LBB }\end{array}$ \\
\hline M & 3,7 & 3,3 & 3 & 2,8 & 1 & 1,6 \\
\hline S & 3,6 & 3,1 & 3 & 2,7 & 1 & 1,7 \\
\hline
\end{tabular}

In the results shown in Tabs. 2, 3 and 4, experts took into account changes in colors and tonal values based on the simultaneous and the memory visual comparison. High consistency of the results of simultaneous and memory comparison confirms the qualitative ability of experts, and high consistency of the results (in the criteria of the color, tone values, saturation and the corrective filters applied) and their acceptability as the expert group. Memory comparison can be considered closer to the real conditions of consumption of the photographic images realized on web pages (and in general) because consumers generally do not directly compare photographic images realized on the different devices and output units. This comparison in the expert group also gives a slightly higher tolerance of color and tone values changes, regardless the degradation method, but generally, the method of visual comparison in the expert group did not significantly affect the estimation of the degraded photographic images.

Tab. 2 shows that experts accept the change of $1 / 3$ of the lens aperture as the limit change, i.e. the degraded tone values and colors are considered acceptable, and larger changes are not. The total exposure increase of $1 / 3$ of the lens aperture is considered completely acceptable, and the decrease of $1 / 3$ of the lens aperture is considered acceptable. Tab. 3 shows that the increase and decrease in saturation within \pm 10 experts estimate as acceptable, but the decrease in the saturation for the maximum observed value -15 is estimated as acceptable as well. This can be explained by the specificity of the portrait as the motif often realized in the black-and-white photographic system, and experts estimated the approach to this system as acceptable. Skin color primarily determines the perception of the portrait photographic image, so its proximity to the achromatic point should be taken into account. For other photographic motifs, in terms of saturation, potentially different results can be expected.

The general acceptability of the color temperature increase, i.e. simulation of warm corrective filters, and extremely low acceptability of cold corrective filters (Tab. 4) coincides with the previous research $[4,13]$ which considers the increase of the $\mathrm{R}$ channel as more acceptable than increase of the B channel. These results should be taken into account in the calibration of output units, and in defining the light consumption conditions.

Tabs. 5-11 show the $L^{*}, a *, b *$ values of the standard skin colors, achromatic colors, and average colors of the Adobe Camera Raw color table for the color table defined as the original (Tab. 5) and the degraded color tables (Tabs. 611). Tabs. 6-11 show the total color differences expressed as $\Delta \mathrm{E}_{00}$ in the relation to the defined original for the observed colors.

Table $5 L^{*}, a^{*}, b^{*}$ values for original color table

\begin{tabular}{|c|c|c|c|c|}
\hline Color & Phase & $\mathrm{L}^{*}$ & $\mathrm{a}^{*}$ & $\mathrm{~b}^{*}$ \\
\hline Dark skin (DS) & Original & 38 & 14 & 16 \\
\hline Light skin (LS) & Original & 67 & 17 & 18 \\
\hline White (W) & Original & 96 & 0 & 0 \\
\hline Neutral 8 & Original & 81 & 0 & 0 \\
\hline Neutral 6.5 & Original & 65 & 0 & 0 \\
\hline Neutral 5 & Original & 51 & 0 & 0 \\
\hline Neutral 3.5 & Original & 35 & 0 & 0 \\
\hline Black (K) & Original & 20 & 0 & 0 \\
\hline Average color & Original & 56,8 & 4 & 4,3 \\
\hline
\end{tabular}

According to previous research results, the limit of $\Delta \mathrm{E}_{00}$ 6 is defined as the acceptable limit for photographic images in real conditions. The change in skin colors by increasing and decreasing the exposure by $1 / 3$ of the lens aperture can be taken as acceptable (Tabs. 6 and 7). Tabs. 6 and 7 show that the change of the exposure for $1 / 3$ of the lens aperture, the observed colors change is within the limits of acceptability, which coincides with the experts' estimations (Tab. 2). In this case, the simulation of the change in exposure primarily affects the lightness (L-value), affecting the tonal values, which in achromatic colors and colors close 
to achromatic colors can be considered as the primer acceptability modulator [4].

Table 6 Color change values by simulating exposure increase

\begin{tabular}{|c|c|c|c|c|c|}
\hline Color & Exposure & $\mathrm{L}^{*}$ & $\mathrm{a}^{*}$ & $\mathrm{~b}^{*}$ & $\Delta E_{00}$ \\
\hline Dark skin (DS) & $+0,33$ & 38 & 14 & 16 & 3,8196 \\
\hline Light skin (LS) & $+0,33$ & 67 & 17 & 18 & 5,4258 \\
\hline White (W) & $+0,33$ & 96 & 0 & 0 & 2,3298 \\
\hline Neutral 8 & $+0,33$ & 81 & 0 & 0 & 5,2605 \\
\hline Neutral 6.5 & $+0,33$ & 65 & 0 & 0 & 5,4471 \\
\hline Neutral 5 & $+0,33$ & 51 & 0 & 0 & 3,9022 \\
\hline Neutral 3.5 & $+0,33$ & 35 & 0 & 0 & 3,3773 \\
\hline Black (K) & $+0,33$ & 20 & 0 & 0 & 1,3987 \\
\hline Average color & - & 56,8 & 4 & 4,3 & 3,870125 \\
\hline Color & Exposure & $\mathrm{L}^{*}$ & $\mathrm{a}^{*}$ & $\mathrm{~b}^{*}$ & \\
\hline Dark skin (DS) & $+0,66$ & 47 & 17 & 20 & 8,5764 \\
\hline Light skin (LS) & $+0,66$ & 81 & 20 & 21 & 10,5264 \\
\hline White (W) & $+0,66$ & 100 & 0 & 0 & 2,3298 \\
\hline Neutral 8 & $+0,66$ & 97 & 0 & 0 & 10,119 \\
\hline Neutral 6.5 & $+0,66$ & 80 & 0 & 0 & 10,4582 \\
\hline Neutral 5 & $+0,66$ & 62 & 0 & 0 & 10,1821 \\
\hline Neutral 3.5 & $+0,66$ & 43 & 0 & 0 & 6,9393 \\
\hline Black (K) & $+0,66$ & 25 & 0 & 0 & 3,5533 \\
\hline Average color & - & 66,8 & 4,6 & 5,1 & 7,8355625 \\
\hline Color & Exposure & $\mathrm{L}^{*}$ & $\mathrm{a}^{*}$ & $\mathrm{~b}^{*}$ & $\Delta E_{00}$ \\
\hline Dark skin (DS) & +1 & 52 & 18 & 21 & 13,6244 \\
\hline Light skin (LS) & +1 & 87 & 14 & 20 & 14,626 \\
\hline White (W) & +1 & 100 & 0 & 0 & 2,3298 \\
\hline Neutral 8 & +1 & 100 & 0 & 0 & 11,8466 \\
\hline Neutral 6.5 & +1 & 87 & 0 & 0 & 15,0867 \\
\hline Neutral 5 & +1 & 68 & 0 & 0 & 15,0585 \\
\hline Neutral 3.5 & +1 & 47 & 0 & 0 & 10,7057 \\
\hline Black (K) & +1 & 25 & 0 & 0 & 3,5533 \\
\hline Average color & - & 70,6 & 4 & 5,1 & 10,853875 \\
\hline
\end{tabular}

Table 7 Color change values by simulating exposure decrease

\begin{tabular}{|c|c|c|c|c|c|}
\hline Color & Exposure & $\mathrm{L}^{*}$ & $\mathrm{a}^{*}$ & $\mathrm{~b}^{*}$ & $\Delta E_{00}$ \\
\hline Dark skin (DS) & $-0,33$ & 34 & 13 & 15 & 3,4271 \\
\hline Light skin (LS) & $-0,33$ & 61 & 15 & 16 & 5,2054 \\
\hline White (W) & $-0,33$ & 88 & 0 & 0 & 4,9187 \\
\hline Neutral 8 & $-0,33$ & 74 & 0 & 0 & 4,9746 \\
\hline Neutral 6.5 & $-0,33$ & 60 & 0 & 0 & 5,0659 \\
\hline Neutral 5 & $-0,33$ & 46 & 0 & 0 & 4,9645 \\
\hline Neutral 3.5 & $-0,33$ & 31 & 0 & 0 & 3,2087 \\
\hline Black (K) & $-0,33$ & 18 & 0 & 0 & 1,3696 \\
\hline Average color & - & 51,5 & 3,5 & 3,875 & 4,1418125 \\
\hline Color & Exposure & $\mathrm{L}^{*}$ & $\mathrm{a}^{*}$ & $\mathrm{~b}^{*}$ & \\
\hline Dark skin (DS) & $-0,66$ & 31 & 11 & 14 & $6 E_{00}$ \\
\hline Light skin (LS) & $-0,66$ & 55 & 14 & 16 & 10,604 \\
\hline White (W) & $-0,66$ & 80 & 0 & 0 & 10,2165 \\
\hline Neutral 8 & $-0,66$ & 67 & 0 & 0 & 10,3404 \\
\hline Neutral 6.5 & $-0,66$ & 54 & 0 & 0 & 10,5547 \\
\hline Neutral 5 & $-0,66$ & 42 & 0 & 0 & 8,7179 \\
\hline Neutral 3.5 & $-0,66$ & 28 & 0 & 0 & 5,513 \\
\hline Black (K) & $-0,66$ & 51 & 0 & 0 & 2,7112 \\
\hline Average color & - & 51 & 3,1 & 3,6 & 8,1043875 \\
\hline Color & Exposure & $\mathrm{L}^{*}$ & $\mathrm{a}^{*}$ & $\mathrm{~b}^{*}$ & $\Delta E_{00}$ \\
\hline Dark skin (DS) & -1 & 28 & 12 & 12 & 8,4224 \\
\hline Light skin (LS) & -1 & 50 & 13 & 14 & 15,5731 \\
\hline White (W) & -1 & 73 & 0 & 0 & 15,1995 \\
\hline Neutral 8 & -1 & 61 & 0 & 0 & 15,2895 \\
\hline Neutral 6.5 & -1 & 49 & 0 & 0 & 15,5021 \\
\hline Neutral 5 & -1 & 37 & 0 & 0 & 13,0577 \\
\hline Neutral 3.5 & -1 & 25 & 0 & 0 & 7,7353 \\
\hline Black (K) & -1 & 14 & 0 & 0 & 4,0255 \\
\hline Average color & - & 42,1 & 3,1 & 3,2 & 11,8506375 \\
\hline
\end{tabular}

Table 8 Color change values by simulating saturation increase

\begin{tabular}{|c|c|c|c|c|c|}
\hline Color & Saturation & $\mathrm{L}^{*}$ & $\mathrm{a}^{*}$ & $\mathrm{~b}^{*}$ & $\Delta E_{00}$ \\
\hline Dark skin (DS) & +5 & 38 & 14 & 17 & 0,6694 \\
\hline Light skin (LS) & +5 & 67 & 18 & 19 & 0,6815 \\
\hline White (W) & +5 & 96 & 0 & 0 & 0 \\
\hline Neutral 8 & +5 & 81 & 0 & 0 & 0 \\
\hline Neutral 6.5 & +5 & 66 & 0 & 0 & 0 \\
\hline Neutral 5 & +5 & 51 & 0 & 0 & 0 \\
\hline Neutral 3.5 & +5 & 35 & 0 & 0 & 0 \\
\hline Black (K) & +5 & 20 & 0 & 0 & 0 \\
\hline Average color & - & 56,8 & 4 & 4,5 & 0,1688625 \\
\hline Color & Saturation & $\mathrm{L}^{*}$ & $\mathrm{a}^{*}$ & $\mathrm{~b}^{*}$ & $\Delta E_{00}$ \\
\hline Dark skin (DS) & +10 & 38 & 16 & 18 & 1,487 \\
\hline Light skin (LS) & +10 & 67 & 18 & 20 & 1,1113 \\
\hline White (W) & +10 & 96 & 0 & 0 & 0 \\
\hline Neutral 8 & +10 & 81 & 0 & 0 & 0 \\
\hline Neutral 6.5 & +10 & 66 & 0 & 0 & 0 \\
\hline Neutral 5 & +10 & 51 & 0 & 0 & 0 \\
\hline Neutral 3.5 & +10 & 35 & 0 & 0 & 0 \\
\hline Black (K) & +10 & 20 & 0 & 0 & 0 \\
\hline Average color & - & 56,8 & 4,3 & 4,8 & 0,3247875 \\
\hline & & & & & \\
\hline Color & Saturation & $\mathrm{L}^{*}$ & $\mathrm{a}^{*}$ & $\mathrm{~b}^{*}$ & $\Delta E_{00}$ \\
\hline Dark skin (DS) & +15 & 38 & 17 & 19 & 2,1822 \\
\hline Light skin (LS) & +15 & 67 & 20 & 21 & 1,9698 \\
\hline White (W) & +15 & 96 & 0 & 0 & 0 \\
\hline Neutral 8 & +15 & 81 & 0 & 0 & 0 \\
\hline Neutral 6.5 & +15 & 66 & 0 & 0 & 0 \\
\hline Neutral 5 & +15 & 51 & 0 & 0 & 0 \\
\hline Neutral 3.5 & +15 & 35 & 0 & 0 & 0 \\
\hline Black (K) & +15 & 20 & 0 & 0 & 0 \\
\hline Average color & - & 56,7 & 4,6 & 5 & 0,519 \\
\hline & & & & \\
\hline
\end{tabular}

Table 9 Color change values by simulation by saturation decrease

\begin{tabular}{l|c|c|c|c|c} 
Color & Saturation & $\mathrm{L}^{*}$ & $\mathrm{a}^{*}$ & $\mathrm{~b}^{*}$ & $\Delta E_{00}$ \\
\hline
\end{tabular}

\begin{tabular}{|c|c|c|c|c|c|}
\hline Color & Saturation & $\mathrm{L}^{*}$ & $\mathrm{a}^{*}$ & $\mathrm{~b}^{*}$ & $\Delta E_{00}$ \\
\hline Dark skin (DS) & -5 & 38 & 14 & 16 & 0 \\
\hline Light skin (LS) & -5 & 67 & 16 & 17 & 0,7092 \\
\hline White (W) & -5 & 96 & 0 & 0 & 0 \\
\hline Neutral 8 & -5 & 81 & 0 & 0 & 0 \\
\hline Neutral 6.5 & -5 & 66 & 0 & 0 & 0 \\
\hline Neutral 5 & -5 & 51 & 0 & 0 & 0 \\
\hline Neutral 3.5 & -5 & 35 & 0 & 0 & 0 \\
\hline Black (K) & -5 & 20 & 0 & 0 & 0 \\
\hline Average color & - & 56,7 & 3,8 & 4,1 & 0,08865 \\
\hline Color & Saturation & $\mathrm{L}^{*}$ & $\mathrm{a}^{*}$ & $\mathrm{~b}^{*}$ & $\Delta E_{00}$ \\
\hline Dark skin (DS) & -10 & 38 & 13 & 15 & 0,7965 \\
\hline Light skin (LS) & -10 & 67 & 15 & 15 & 1,8522 \\
\hline White (W) & -10 & 96 & 0 & 0 & 0 \\
\hline Neutral 8 & -10 & 81 & 0 & 0 & 0 \\
\hline Neutral 6.5 & -10 & 66 & 0 & 0 & 0 \\
\hline Neutral 5 & -10 & 51 & 0 & 0 & 0 \\
\hline Neutral 3.5 & -10 & 35 & 0 & 0 & 0 \\
\hline Black (K) & -10 & 20 & 0 & 0 & 0 \\
\hline Average color & - & 56,7 & 3,5 & 3,8 & 0,3310875 \\
\hline & & & & & \\
\hline Color & Saturation & $\mathrm{L}^{*}$ & $\mathrm{a}^{*}$ & $\mathrm{~b}^{*}$ & $\Delta E_{00}$ \\
\hline Dark skin (DS) & -15 & 39 & 12 & 13 & 2,1954 \\
\hline Light skin (LS) & -15 & 67 & 14 & 15 & 2,2198 \\
\hline White (W) & -15 & 96 & 0 & 0 & 0 \\
\hline Neutral 8 & -15 & 81 & 0 & 0 & 0 \\
\hline Neutral 6.5 & -15 & 66 & 0 & 0 & 0 \\
\hline Neutral 5 & -15 & 51 & 0 & 0 & 0 \\
\hline Neutral 3.5 & -15 & 35 & 0 & 0 & 0 \\
\hline Black (K) & -15 & 20 & 0 & 0 & 0 \\
\hline Average color & - & 56,8 & 3,25 & 3,5 & 0,5519 \\
\hline & & & & \\
\hline
\end{tabular}


Table 10 Color change values by simulating a corrective filter

\begin{tabular}{|c|c|c|c|c|c|}
\hline Color & Filter & $\mathrm{L}^{*}$ & $\mathrm{a}^{*}$ & $\mathrm{~b}^{*}$ & $\Delta E_{00}$ \\
\hline Dark skin (DS) & WF 81 & 38 & 18 & 22 & 3,4978 \\
\hline Light skin (LS) & WF 81 & 67 & 21 & 28 & 5,0751 \\
\hline White (W) & WF 81 & 97 & 2 & 6 & 5,858 \\
\hline Neutral 8 & WF 81 & 81 & 10 & 18 & 15,2811 \\
\hline Neutral 6.5 & WF 81 & 66 & 8 & 15 & 13,3845 \\
\hline Neutral 5 & WF 81 & 51 & 7 & 11 & 11,3181 \\
\hline Neutral 3.5 & WF 81 & 35 & 5 & 9 & 9,2675 \\
\hline Black (K) & WF 81 & 20 & 4 & 6 & 7,1239 \\
\hline Average color & - & 56,8 & 9,3 & 14,3 & 8,85075 \\
\hline Color & Filter & L$^{*}$ & $\mathrm{a}^{*}$ & $\mathrm{~b}^{*}$ & \\
\hline Dark skin (DS) & WF 85 & 96 & 3 & 6 & 6,4165 \\
\hline Light skin (LS) & WF 85 & 81 & 5 & 6 & 7,8959 \\
\hline White (W) & WF 85 & 96 & 3 & 6 & 6,4165 \\
\hline Neutral 8 & WF 85 & 81 & 5 & 6 & 7,8959 \\
\hline Neutral 6.5 & WF 85 & 66 & 3 & 5 & 5,8421 \\
\hline Neutral 5 & WF 85 & 51 & 3 & 4 & 5,3022 \\
\hline Neutral 3.5 & WF 85 & 35 & 2 & 3 & 3,8729 \\
\hline Black (K) & WF 85 & 20 & 1 & 3 & 3,1187 \\
\hline Average color & - & 56,7 & 6,5 & 8,25 & 4,4541375 \\
\hline Color & Filter & L* & $\mathrm{a}^{*}$ & $\mathrm{~b}^{*}$ & \\
\hline Dark skin (DS) & WF LBA & 38 & 18 & 19 & 2,1276 \\
\hline Light skin (LS) & WF LBA & 67 & 21 & 24 & 3,2632 \\
\hline White (W) & WF LBA & 97 & 3 & 5 & 5,8719 \\
\hline Neutral 8 & WF LBA & 81 & 8 & 12 & 12,2621 \\
\hline Neutral 6.5 & WF LBA & 66 & 7 & 11 & 11,3181 \\
\hline Neutral 5 & WF LBA & 51 & 6 & 8 & 9,4696 \\
\hline Neutral 3.5 & WF LBA & 35 & 5 & 6 & 7,8959 \\
\hline Black (K) & WF LBA & 20 & 3 & 3 & 4,8214 \\
\hline Average color & - & 56,9 & 8,9 & 11 & 7,128725 \\
\hline
\end{tabular}

Table 11 Color change values by simulating a corrective filter

\begin{tabular}{|c|c|c|c|c|c|}
\hline Color & Filter & $\mathrm{L}^{*}$ & $\mathrm{a}^{*}$ & $\mathrm{~b}^{*}$ & $\Delta E_{00}$ \\
\hline Dark skin (DS) & CF LBB & 37 & 18 & 0 & 12,5961 \\
\hline Light skin (LS) & CF LBB & 66 & 23 & -4 & 16,3963 \\
\hline White (W) & CF LBB & 98 & 1 & -3 & 3,3328 \\
\hline Neutral 8 & CF LBB & 80 & 13 & -20 & 17,0388 \\
\hline Neutral 6.5 & CF LBB & 65 & 11 & -17 & 15,4064 \\
\hline Neutral 5 & CF LBB & 50 & 9 & -15 & 13,8787 \\
\hline Neutral 3.5 & CF LBB & 34 & 7 & -11 & 11,3475 \\
\hline Black (K) & CF LBB & 19 & 5 & -9 & 9,293 \\
\hline Average color & - & 56,1 & 10,9 & $-9,9$ & 12,4112 \\
\hline Color & Filter & L* & $\mathrm{a}^{*}$ & $\mathrm{~b}^{*}$ & \\
\hline Dark skin (DS) & CF 82 & 37 & 18 & 0 & 3,3328 \\
\hline Light skin (LS) & CF 82 & 66 & 23 & -4 & 17,0388 \\
\hline White (W) & CF 82 & 98 & 1 & -3 & 15,3847 \\
\hline Neutral 8 & CF 82 & 80 & 13 & -20 & 13,8787 \\
\hline Neutral 6.5 & CF 82 & 66 & 11 & -17 & 11,3475 \\
\hline Neutral 5 & CF 82 & 50 & 9 & -15 & 9,293 \\
\hline Neutral 3.5 & CF 82 & 34 & 7 & -11 & 3,3328 \\
\hline Black (K) & CF 82 & 19 & 5 & -9 & 17,0388 \\
\hline Average color & - & 56,2 & 10,8 & $-9,8$ & 12,358175 \\
\hline & & & & & \\
\hline Color & Filter & L* & $\mathrm{a}^{*}$ & $\mathrm{~b}^{*}$ & $\Delta E_{00}$ \\
\hline Dark skin (DS) & CF 80 & 38 & 17 & 11 & 4,9309 \\
\hline Light skin (LS) & CF 80 & 67 & 21 & 11 & 6,4205 \\
\hline White (W) & CF 80 & 97 & 1 & -4 & 3,9418 \\
\hline Neutral 8 & CF 80 & 80 & 11 & -1 & 12,0538 \\
\hline Neutral 6.5 & CF 80 & 65 & 8 & 0 & 9,4801 \\
\hline Neutral 5 & CF 80 & 50 & 7 & -1 & 8,5804 \\
\hline Neutral 3.5 & CF 80 & 34 & 5 & -1 & 6,5165 \\
\hline Black (K) & CF 80 & 20 & 3 & -2 & 4,4331 \\
\hline Average color & - & 56,4 & 9,1 & 1,6 & 7,04463 \\
\hline
\end{tabular}

Tabs. 8 and 9 show the changes in the observed colors by changing the saturation. Increase and decrease in saturation by the value of 5 was estimated as completely acceptable (Tab. 3). The measured values show the changes that are assessed as visually imperceptible ( $\Delta \mathrm{E}_{00} 0.2$ or less). It should be emphasized that Tabs. 8 and 9 indicate small overall color differences of degraded photographic images compared to the original by changing the saturation within the observed limits and that the saturation change of \pm 15 still gives acceptable differences $\left(\Delta E_{00}<3\right)$ even for the consumption by direct comparison, although experts estimated the change in saturation +15 as unacceptable (Tab. 2 ). This can be explained by the specifics of skin color as the primer modulator of portrait photographic image perception.

Tab. 10 indicates that the simulation of the warm corrective filter WF 85 application changes the standard skin color above the limits of acceptability $\left(\Delta E_{00}>6\right)$ but, due to the perception of the warmer skin color as the healthier, experts estimate this degradation as acceptable as well. The application of the cold corrective filters (Tab. 11) changes colors more markedly, i.e. in the observed cases $\Delta \mathrm{E}_{00}$ changes are larger than with the application of warm corrective filters (Tab. 10). As the acceptable degradation limit for cold corrective filters, only the change by the CF 80 filter can be taken, which was also confirmed as acceptable by the experts' estimation.

Tabs. 12, 13, and 14 show the acceptability of reducing the sharpness of the photographic image - Tab. 12 by expert evaluations, Tab. 13 the ability of line separation by degrading of sharpness simulation, and Tab. 14 by reducing the fineness of JPEG records. Tabs. 13 and 14 show the $\mathrm{LW} / \mathrm{PH}$ values according to ISO 12233.

Table 12 Degradation by sharpness decreases and fineness of the record change the expert evaluation

\begin{tabular}{|c|c|c|c|c|c|c|c|c|c|}
\hline Method & 10 & 20 & 30 & 40 & 50 & 60 & L & M & Max \\
\hline M & 3,8 & 4 & 3 & 2,5 & 1 & 1 & 4 & 4 & 4 \\
\hline S & 3,7 & 4 & 3 & 2,4 & 1 & 1 & 4 & 4 & 4 \\
\hline
\end{tabular}

Table 13 Degradation by sharpness decrease - line separation according to table

\begin{tabular}{|c|c|c|c|c|c|c|c|}
\hline Direction & Original & 10 & 20 & 30 & 40 & 50 & 60 \\
\hline Horizontal & 1400 & 1000 & 600 & 300 & 150 & - & - \\
\hline Vertical & 1500 & 1200 & 600 & 300 & 150 & - & - \\
\hline
\end{tabular}

According to the experts, the degradation of sharpness for the value of 40 is the limit of acceptability, and the value of 50 is unacceptable. According to Tab. 13, this degradation limit gives the line separation ability of $150 \mathrm{LW} / \mathrm{PH}$. Tab. 12 also shows that experts consider all observed degradations by reducing the fineness of JPEG records to be completely acceptable, which is confirmed by the practically unchanged ability of line separation according to ISO 12233 shown in Tab. 14.

Table 14 Degradation by fineness of the record change for Low 1 , Medium 5 , Height 8, Maximum 12 - line separation according to table 12233

\begin{tabular}{|c|c|c|c|c|c|}
\hline Direction & Original & 12 & 8 & 5 & 1 \\
\hline Horizontal & 1400 & 1400 & 1400 & 1400 & 1400 \\
\hline Vertical & 1500 & 1500 & 1500 & 1500 & 1400 \\
\hline
\end{tabular}

\section{CONCLUSION}

Research analysis confirmed that the Internet is recognized as a place of tourist promotion and that certain 
elements affect the quality of the website in terms of information, ease of access to certain content. The selected page is adapted to the use of multiple search engines. They are well connected with social networks, although it has been noticed that the potential of Instagram is not used enough. Multilingualism is well represented, with dominant English. Visual intelligibility is based on the consistent use of the principles of contrast, balance, rhythm, composition and unity. On the symbolic level, the content is most often communicated through color. The perception of the Republic of Croatia based on the research results of this element show that it is still perceived as a destination of the sea and the sun. All analyzed destinations have a pronounced visual element, and what is missing is a complete visual story of the Republic of Croatia, which can be changed following the strategic national guidelines for web promotion. When designing a website, the authors suggest the implementation of a central photography and a larger number of photographic images, logos with the use of color that follows a coherent approach to tourism promotion at the national level. Scientific research based on the eye-tracker analysis indicates the importance of implementing facial photographic image into touristic web page design. Research results proved facial photographic image to be the most important element of entry, based on the results of speed of observation and length of focus on individual content, measuring time to first fixation, total duration of fixation, fixation count and visit duration. The authors suggest the inclusion of facial photographic image as an integral element in the creation of tourist websites. The authors propose the application of the conducted research methods based on deductive sequence for predicting future scientific researches. Based on the scientific research of facial photographic image's technical characteristics, the authors propose certain guidelines regarding the quality control of the display and experience related to the characteristics of color, sharpness, saturation, using simulation and degradation of facial photographic image that can positively reflect on tourism development.

Based on the study of the acceptability of the degradation of a portrait photographic image that corresponds to the technical website's photographic images characteristics, it is concluded that the degradation of tones and colors due to simulation of exposure change $1 / 3$ of the lens aperture is in the limit of acceptability. This is confirmed by expert estimation, determination of the total color difference $\Delta E_{00}$ and the changes in height (i.e. L value) observing the skin color as the dominant in the portrait photographic image analysis. The change in saturation within limits of \pm 10 can be defined as completely acceptable saturation degradation, based on the experts' estimation and the determination of the total color difference. The portrait is the photographic motif in which the perception of the photographic image primarily depends on the reproduction of skin color. Near the achromatic point, experts' estimation confirms even greater tolerance in saturation decrease, but this conclusion cannot be considered as universal for other photographic motifs of websites in the function of tourism promotion and development.
Expert estimation and determination of the total color difference indicate a higher tolerance for the simulation of changes by warm than cold corrective filter application, which coincides with previous research of portrait photographic images indicating the higher tolerance of increasing the value of $\mathrm{R}$, than $\mathrm{B}$ recording channels. This conclusion, apart from being an indicator of degradation tolerance, can also be used in the output unit calibration and light consumption conditions.

Regarding the tolerance of degradation of the portrait photographic image used for the realization of web pages in the function of promotion and development of tourism, the limit of acceptability in decrease in sharpness the value of 40 is defined, while for the fully acceptable degradation the limit of value 20 can be defined. It means reducing the ability to separate lines from 1400 (horizontal), 1500 (vertical) LW / PH for original to 600 (horizontal) 600 (vertical) LW / PH for value of degradation 20 and 150 (horizontal) 150 (vertical) for value of degradation 40. Reducing the fineness of JPEG format recording did not affect the acceptability of the sharpness of the observed portrait photographic images, which can be used in optimizing records for the realization of websites in the function of tourism promotion and development.

\section{REFERENCES}

[1] Ministarstvo turizma Republike Hrvatske - Strategija razvoja turizma. https://mint.gov.hr/strategija-razvoja-turizma11411/11411 (Accessed: 11.02.2021) (in Croatian)

[2] Mikota, M. (2016). Fotografija - Tehnički i semantičkosintaktički izazovi medija u novom okruženju. Tiskarstvo \& Dizajn 2016, 175-178. (in Croatian)

[3] Periša, M., Mrvac, N. \& Mikota, M. (2015). The visual grammar of photographic images produced by media convergence. Communication Management Forum Proceedings Zagreb, Edward Bernays, 647-667.

[4] Mikota, M., Brozović, M., \& Pavlović, I. (2008). Kvalitetafotografske prezentacije modnih noviteta u medijima vanjskog oglašavanja. Tekstil: časopis za tekstilnu tehnologiju konfekciju, 57(9), 457-464. (in Croatian)

[5] Ahtik, J. \& Starešinič, M. (2017). Eye movement analysis of image quality parameters compared to subjective image quality assessment. Tehnički vjesnik, 24(6), 1833-1839. https://doi.org/10.17559/TV-20161213185321

[6] Mohammadi, P., Ebrahimi-Moghadam, A., \& Shirani S. (2014). Subjective and objective quality assessment of image. Majlesi Journal of Electrical Engineering, 9(1), 55-83.

[7] Pavlović, I., Mikota, M., \& Mrvac, N. (2009). Exposure Correction in Digital Portrait Photography Taken with the Nitraphot Lighting. DAAAM Symposium "Inteligent Manifacturing \& Automation: Focus on Theory, Practice and Education". DAAAM International, 1449-1450.

[8] Pilarczyk, J., \& Kuniecki, M. (2014). Emotional content of an image attracts attention more than visually salient features in various signal-to-noise ratio conditions. Journal of Vision, 14(12), 1-19. https://doi.org/10.1167/14.12.4

[9] Ha, J., Byon, Y.-J., Cho, C.-S., \& Seong, P. (2018). EyeTracking Studies Based on Attentional-Resource Effectiveness and Insights into Future Research. Nuclear Technology, 202, 110. https://doi.org/10.1080/00295450.2018.1428003 
[10] Erhu, Z., Jia, Y., Yajun, C., \& Yang, Y., (2010). A study of image color quality evaluation based on S-CIELAB. The $3^{\text {rd }}$ International Congress on Image and Signal Processing, 3. https://doi.org/10.1109/CISP.2010.5646866

[11] Sharma, G., Wu, W., \& Dalal, E., N. (2005). The CIEDE2000 color difference formula: implementation notes, supplementary test dana and mathematical observations. Journal Color Research \& Application, 30(1) 21-30. https://doi.org/10.1002/col.20070

[12] ISO 3664: 2009 Graphic technology and photography Viewing conditions https://www.iso.org/standard/43234.html

[13] Mikota, M., Matijević, M., \& Pavlović, I. (2016). Colour reproduction analysis of portrait photography in crossmedia system: image on the computer monitorelectrophotographic printing. Imaging Science Journal, 64(6), 299-304. https://doi.org/10.1080/13682199.2016.1184875

\section{Authors' contacts:}

\section{Petra Ptiček}

University of Zagreb, Faculty of Graphic Arts,

Getaldićeva 2,

10000 Zagreb, Croatia

E-mail: pticekpetra@gmail.com

Ivana Žganjar, $\mathrm{PhD}$

University of Zagreb, Faculty of Graphic Arts,

Getaldićeva 2,

10000 Zagreb, Croatia

E-mail: ivana.zganjar@grf.unizg.hr

Miroslav Mikota, PhD, Assistant Professor

(Corresponding author)

University of Zagreb, Faculty of Graphic Arts,

Getaldićeva 2.

10000 Zagreb, Croatia

E-mail: miroslav.mikota@grf.unizg.hr

Mile Matijević, PhD, Assistant Professor

University of Zagreb, Faculty of Graphic Arts,

Getaldićeva 2,

10000 Zagreb, Croatia

E-mail: mile.matijevic@grf.unizg.hr 